\title{
Application of rapid artificial cardiac pacing in thoracic endovascular aortic repair in aged patients
}

This article was published in the following Dove Press journal:

Clinical Interventions in Aging

27 December 2013

Number of times this article has been viewed

\section{Jun Chen ${ }^{1, *}$ \\ Wenhui Huang2,* \\ Songyuan Luo ${ }^{2, *}$ \\ Dahao Yang' \\ Zhengrong $\mathrm{Xu}^{\prime}$ \\ Jianfang Luo ${ }^{2}$}

'Department of Angiocardiopathy, Affiliated Baoan Hospital of Southern Medical University, Shenzhen City, People's Republic of China; ${ }^{2}$ Department of Cardiology, Guangdong Cardiovascular Institute, Guangdong General Hospital, Guangdong Academy of Medical Sciences, Guangzhou, People's Republic of China

*These authors contributed equally to this work
Correspondence: Zhengrong $\mathrm{Xu}$ Department of Angiocardiopathy, Affiliated Baoan Hospital of Southern Medical University, Shenzhen City, Guangdong 5I8I0I, People's Republic of China Email xuzhengrong_smu@163.com

Jianfang Luo

Department of Cardiology, Guangdong Cardiovascular Institute, Guangdong General Hospital, Guangdong Academy of Medical Sciences, Guangzhou, Guangdong 510080, People's Republic of China Email henryjfl@tom.com
Objective: To compare the safety, efficacy, and impact on stent graft positioning between rapid artificial cardiac pacing (RACP), induced hypotension and sodium nitroprusside (SNP) induced hypotension during thoracic endovascular aortic repair (TEVAR) for Stanford B aortic dissection.

Methods: One hundred and sixty-eight patients, who were diagnosed with Stanford B aortic dissection and who underwent selective TEVAR in Guangdong General Hospital and the People's Hospital of Baoan District, Shenzhen, People's Republic of China, were enrolled in this study. Patients were randomly divided into a RACP group $(n=77)$ and a SNP group $(n=91)$. During localization and deployment of the stent graft, hypotension was induced by RACP or intravenous SNP, according to randomization. Hemodynamics, landing precision (deviation from planned placement site), duration of procedure, renal function, neurocognitive function, and incidence of endoleaks and paraplegia/hemiplegia were compared. Except for methods of inducing hypotension, TEVAR was performed according to the same protocol in each group.

Results: RACP was successfully performed in all patients assigned to the RACP group. Compared with the SNP group, blood pressure was significantly lower ( $43 \pm 5$ versus $81 \pm 6 \mathrm{mmHg}$, $P=0.003$ ) and the restoration time of blood pressure and the operation duration were significantly shorter ( $7 \pm 2$ versus $451 \pm 87$ seconds, $P<0.001 ; 87 \pm 15$ versus $106 \pm 18$ minutes, $P<0.001$, respectively) in the RACP group. Stent graft localization/deployment was more precise in the RACP group ( $2 \pm 1$ versus $5 \pm 2 \mathrm{~mm}, P<0.001$ ). Compared to baseline, there was no significant change after TEVAR in either group in regard to renal function, neurocognitive function, and incidence of endoleaks and paraplegia/hemiplegia.

Conclusion: RACP can be safely applied to patients undergoing TEVAR for Stanford B dissection. RACP can shorten the operation duration and facilitate precise graft localization/ deployment.

Keywords: Stanford B aortic dissection, endovascular repair, rapid artificial cardiac pacing, precise graft deployment

\section{Introduction}

Aortic dissection is a critical condition of the cardiovascular system with fast onset and high mortality. In the People's Republic of China, the incidence of aortic dissection, which poses a tremendous threat to people's health, tends to rise yearly. ${ }^{1}$ Traditionally, aortic dissection was treated with aortic artificial vascular graft under thoracotomy, which is more invasive and prone to more severe postoperative complications. Since its successful application by Parodi for abdominal aortic aneurysm in 1991, thoracic endovascular aortic repair (TEVAR) has been increasingly used to treat Stanford B aortic dissection. ${ }^{2}$ Generally, the proximal tears in Stanford B aortic dissection are very close to the origin of 
the left subclavian artery and left common carotid artery, which means the landing zone is limited and precise localization and deployment of the stent graft is required to avoid compromising the blood supply to these side branches. Many methods have been applied to facilitate acute localization and deployment of the stent graft. Among them, medication (eg, sodium nitroprusside [SNP])-induced hypotension is the most widely used, although some disadvantages have been reported..$^{3,4}$ In 2006, the authors first introduced rapid artificial cardiac pacing (RACP) among patients undergoing TEVAR for Stanford $\mathrm{B}$ aortic dissection. The present study aimed to compare the safety and impact of RACP and SNP-induced hypotension in patients undergoing TEVAR for aortic dissection.

\section{Subjects and methods Research design}

This study comprised prospective, randomized, case control research. From September 2007 to February 2009, 197 patients who were diagnosed with Stanford B aortic dissection and who underwent TEVAR were enrolled. The patients were randomly divided into a RACP group $(n=77)$ and a SNP group $(n=91)$. During localization and deployment of the stent graft, hypotension was induced by RACP in patients assigned to the RACP group and by intravenous SNP in those assigned to the SNP group, in an attempt to facilitate precise graft localization/deployment. Apart from the induction method, TEVAR was performed according to the same protocol in each group.

\section{Stents}

Talent ${ }^{\mathrm{TM}}$ (Medtronic, Inc, Minneapolis, MN, USA) and Hercules $^{\mathrm{TM}}$ (Shanghai MicroPort Medical [Group] Co, Ltd, Shanghai, People's Republic of China) stents were used in this study.

\section{Anesthesia}

For all the punctures the local infiltration, lidocaine was administered as local anesthesia before femoral artery puncture. When the puncture site was expanded or the stent graft inserted (approximately $1 \mathrm{~cm}$ ), $10 \mathrm{~mL}$ lidocaine was generally supplied.

\section{Surgical procedure}

The patients were in a supine position after anesthesia, and the left radial artery puncture was made with the Seldinger approach. ${ }^{2,4}$ A $6 \mathrm{~F}$ pigtail catheter was inserted into the ascending aorta for aortic angiography by the following procedure: the catheter was placed into the proximal opening of the left subclavian artery, and the booster was used to expand the aortic arch to the maximum angle (average of $45^{\circ}$ ). Forty milliliters of contrast was injected at the speed of $20 \mathrm{~mL} / \mathrm{second}$ and left for exposure for 10-12 seconds. The real and false lumens, as well as the dissection gap positions, were found, and then the openings of the celiac artery, superior mesenteric artery, and renal artery, within the real and false lumens, were checked.

The Seldinger approach of puncture into the femoral artery was made from the ideal side of the dissection relative to the real lumen, at $1-2 \mathrm{~cm}$ above the bifurcation point between the deep and superficial femoral artery (which was found with computed tomography angiography prior to surgery). Next, the sheath of artery was delivered, and the correct positioning was confirmed with angiography. The loach wire was then passed through the catheter and shown with intermittent imaging to have entered the aortic real lumen. The angiography catheter advanced into sent to the ascending aorta and then the loach wire was replaced by a hard guide wire. The angiography catheter was then removed. The contralateral femoral vein was punctured to implant the temporary pacemaker at the right ventricular apex and the pacemaker adjusted for RACP. The aortic stent graft was passed through to the landing zone of the aortic arch. Hypertension was controlled after correct positioning of the stent was confirmed with angiography.

The landing zone represents the normal artery tissues that are located proximally to the cleft and the hematoma, and was confirmed by main artery computed tomography angiography prior to the surgery. The landing zone requires a distance of $2 \mathrm{~cm}$. Whether the landing zone would cover the opening of the subclavian artery was determined by the distance from the opening to the hematoma - the landing zone may cover the subclavian artery if the distance is too short. The landing zone should not cover the hematoma.

For controlled hypertension, RACP or SNP was employed. For RACP, the temporary pacemaker was adjusted to 180 beats/minute, until the systolic blood pressure dropped to $45 \mathrm{mmHg}$. Then the graft was released section by section, with the bracket naturally open and fixed on the aorta wall. In the SNP group, SNP drugs were intravenously infused, with the blood pressure monitored. The stent was released section by section when the blood pressure dropped below $80 \mathrm{mmHg}$. The SNP drugs were stopped once the stent was fully released.

After the stent was released, angiography was immediately performed to examine the positioning and morphology of the stent and to check for any endoleaks or whether the upper aortic arch branch flowed well. A second thoracic 
aorta angiography was performed to examine the extent of gap closure and check for reduction of endoleaks. Finally, the femoral artery puncture was closed by Perclose stapler suture.

\section{Evaluation of neurocognitive function}

Before and after the operation, neurocognitive dysfunction was evaluated in all patients by the Folstein mini-mental state examination (MMSE) (maximum 30 points). ${ }^{5}$ Patients were divided into four groups: serious cognitive dysfunction (0 17 points); moderate cognitive dysfunction (18 24 points); mild cognitive dysfunction (25 26 points); and normal cognitive function (27 30 points). ${ }^{6,7}$

\section{Precision of deployment}

The distance between the preset anchoring point and the proximal marker of stent graft was measured to evaluate the precision of deployment.

\section{Statistics}

Normally distributed variables were expressed as mean \pm standard deviation, and categorical variables were presented as frequency and percent. Differences of continuous variables were analyzed by Student's $t$-tests, and those of categorical variables by $\chi^{2}$ tests or Fisher's exact test. The Mann-Whitney $\mathrm{U}$ test was applied to analyze abnormal distributed variables like estimated glomerular filtration rate (eGFR) and MMSE scores. A $P$-value $<0.05$ (two-tailed) was considered significant. Analyses were performed with SPSS software (version 17.0; IBM Corporation, Armonk, NY USA).

\section{Results}

Baseline characteristics (eg, age, complications, pharmacotherapy, and basic renal function) were not significantly different between the SNP and RACP groups $(P>0.05)$ (Table 1).

When compared to the patients in the SNP group, the pressure-lowering effect was more significant, the restoration time of blood pressure was shorter, and the stent graft deployment was more precise in patients in the RACP group (Table 2).

In each of the two groups, the cognitive function and renal function were not significantly different before and after the procedure (Table 3 ).

The incidence of endoleak and paraplegia/hemiplegia was not significantly different between the two groups. In both groups, paraplegia/hemiplegia occurred only in cases of long $(20 \mathrm{~cm})$ stent graft implantation (Table 4).
Table I Baseline characteristics of the two groups

\begin{tabular}{|c|c|c|c|}
\hline & $\begin{array}{l}\text { SNP group } \\
(n=91)\end{array}$ & $\begin{array}{l}\text { RACP group } \\
(n=77)\end{array}$ & $P$ \\
\hline Male & $51(56.0)$ & $4 \mathrm{I}(53.2)$ & 0.72 \\
\hline Age, years & $65 \pm 9.2$ & $61 \pm 9.9$ & 0.84 \\
\hline Hypertension & $82(90.1)$ & $67(87.0)$ & 0.53 \\
\hline Diabetes & $10(11)$ & II (I4.3) & 0.52 \\
\hline Lung disease & $19(20.9)$ & $16(20.8)$ & 0.99 \\
\hline Coronary heart disease & $24(26.4)$ & $21(27.3)$ & 0.89 \\
\hline \multicolumn{4}{|l|}{ Drugs } \\
\hline$\beta$-receptor blocker & $88(96.7)$ & $73(94.8)$ & 0.54 \\
\hline ACEi/ARB & 83 (9।.2) & $67(87)$ & 0.38 \\
\hline Venous vasodilator & $84(92.3)$ & 70 (90.9) & 0.74 \\
\hline Diuretics & $25(27.5)$ & $18(23.4)$ & 0.54 \\
\hline$\alpha$-receptor blocker & $4(4.1)$ & $8(8.1)$ & 0.74 \\
\hline Calcium ion antagonist & $52(57.1)$ & $54(70.1)$ & 0.08 \\
\hline \multicolumn{4}{|l|}{ Stent } \\
\hline I4 cm (microinvasive) & $46(50.5)$ & $34(44.2)$ & 0.69 \\
\hline $15 \mathrm{~cm}$ (Medtronic) & $19(20.9)$ & $17(22.1)$ & 0.69 \\
\hline $20 \mathrm{~cm}$ (Medtronic) & $31(28.6)$ & $32(21.4)$ & 0.39 \\
\hline
\end{tabular}

Notes: Data are presented as mean \pm standard deviation or number (percent). Medtronic, Inc, Minneapolis, MN, USA.

Abbreviations: ACEi, angiotensin converting enzyme inhibitor; ARB, angiotensinogen receptor blocker.

\section{Discussion}

Following improvements in stent graft design and manufacturing technique and innovations in the operation method, TEVAR has gradually replaced the traditional aortic replacement as the standard operation method for Stanford B aortic dissection. How to precisely deploy the stent graft at the preset position is critically important for successful operation. The most important factor among the several that influence precise stent graft deployment

Table 2 Hemodynamic indices of the two groups

\begin{tabular}{lccc}
\hline & $\begin{array}{l}\text { SNP group } \\
(\mathbf{n = 9 1 )}\end{array}$ & $\begin{array}{l}\text { RACP group } \\
(\mathbf{n}=\mathbf{7 7})\end{array}$ & $\mathbf{P}$ \\
\hline $\begin{array}{l}\text { Mean arterial pressure } \\
\text { Basic blood pressure } \\
\text { (mmHg) }\end{array}$ & $96 \pm 5$ & $102 \pm 4$ & $>0.05$ \\
$\begin{array}{l}\text { Blood pressure during } \\
\text { the intervention (mmHg) }\end{array}$ & $81 \pm 6$ & $43 \pm 5$ & 0.003 \\
$\begin{array}{l}\text { Blood pressure after the } \\
\text { intervention (mmHg) }\end{array}$ & $97 \pm 4$ & $101 \pm 4$ & $>0.05$ \\
$\begin{array}{l}\text { Restoration time of } \\
\text { blood pressure (seconds) }\end{array}$ & $451 \pm 87$ & $7 \pm 2$ & $<0.001$ \\
$\begin{array}{l}\text { Heart rate } \\
\text { Basic heart rate (bpm) }\end{array}$ & $64 \pm 6$ & $66 \pm 3$ & $>0.05$ \\
$\begin{array}{l}\text { Heart rate during the } \\
\text { intervention (bpm) }\end{array}$ & $72+7$ & $191 \pm 10$ & $<0.001$ \\
$\begin{array}{l}\text { Heart rate after the } \\
\text { intervention (bpm) }\end{array}$ & $65 \pm 4$ & $67 \pm 4$ & $>0.05$ \\
$\begin{array}{l}\text { Operation duration } \\
\text { (minutes) }\end{array}$ & $106 \pm 18$ & $87 \pm 15$ & $<0.001$ \\
$\begin{array}{l}\text { Precision of graft } \\
\text { localization (mm) }\end{array}$ & $5 \pm 2$ & $2 \pm 1$ & $<0.001$ \\
\hline
\end{tabular}

Note: Data are presented as mean \pm standard deviation. 
Table 3 eGFR and cognitive function before and after the operation

\begin{tabular}{|c|c|c|c|c|c|c|}
\hline & \multicolumn{3}{|l|}{ SNP group $(n=91)$} & \multicolumn{3}{|l|}{ RACP group $(n=77)$} \\
\hline & Before the operation & After the operation & $P$ & Before the operation & After the operation & $P$ \\
\hline eGFR & $67(53-75)$ & $65(50-82)$ & $>0.05$ & $65(53-76)$ & $66.5(5 I-76)$ & $>0.05$ \\
\hline Cognitive function & $27(26-29)$ & $27(26-28)$ & $>0.05$ & $27(26-29)$ & $27(26-29)$ & $>0.05$ \\
\hline
\end{tabular}

Note: Data are presented as number (percentage range).

Abbreviations: eGFR, estimated glomerular filtration rate; SNP, sodium nitroprusside; RACP, rapid artificial cardiac pacing.

is the windsock effect: ${ }^{8}$ during stent graft deployment (especially while opening the front end of the stent graft), the blood flow in the aorta is completely blocked by the partly deployed stent graft, which leads to an abrupt increase of blood pressure onto the stent graft, which pushes the stent graft backward.

Lowering pressure during operation is an effective method by which to avoid the adverse influences of the windsock effect. ${ }^{9}$ In general, blood pressure is lowered by the following methods:

1. Manually. The carotid sinus is compressed to stimulate the vagal reflex in order to achieve a pressure-lowering effect. This method is adopted by few doctors, due to the inconstant effect and poor blood pressure control.

2. With medications (eg, SNP, nitroglycerin, and short-acting $\beta$-blockers) ${ }^{4,8}$ The medication approach is employed by most doctors, but still has some disadvantages (eg, individualized dose, unstable pressure-lowering effect, adverse effect on cognitive/renal function, and long restoration time of blood pressure).$^{10}$ Furthermore, it is believed that, even though the medication approach decreases peripheral resistance, it also increases cardiac output, which may subsequently offset its improvement effect on the precise stent graft localization/deployment. ${ }^{8}$

3. By partial right atrial inflow occlusion. ${ }^{11}$ In a normal human body, two-thirds of blood backflow to the right side of the heart comes from the inferior vena cava. If the blood flow from the inferior vena cava is blocked, the cardiac output will be immediately reduced by two-thirds, theoretically, so a pressure-lowering effect could be achieved. This technique has been recently adopted by some American doctors to lower blood pressure, but it is used in only a few centers; its reliability and safety require further investigation.

In the present study, when compared to the traditional pressure-lowering technique via pharmacological modula-

Table 4 Incidence rate of paraplegia and endoleaks

\begin{tabular}{llll}
\hline & SNP group $(\mathbf{n}=91)$ & RACP group $(\mathbf{n}=77)$ & $P$ \\
\hline Paraplegia & $6(6.5)$ & $4(4.9)$ & 0.516 \\
Endoleaks & $7(7.7)$ & $4(4.9)$ & 0.765 \\
\hline
\end{tabular}

Note: Data are presented as number (percent).

Abbreviations: SNP, sodium nitroprusside; RACP, rapid artificial cardiac pacing. tion, RACP could significantly reduce the cardiac output, thus effectively reduce the impact of blood flow on the stent graft, without showing additional side effects. RACP has been routinely applied during percutaneous aortic valve replacement to facilitate precise valve deployment, ${ }^{6}$ without any reported relevant complication from more than 10,000 patients. Therefore, in 2006, our hospital (The Affiliated Baoan Hospital of SMU and Guangdong General Hospital) first applied RACP for TEVAR in China to avoid the windsock effect during stent graft positioning/deployment. If the stent graft jumps forward when the stent graft is partly deployed, the pacing can be stopped temporarily to allow the windsock effect to push the stent graft slightly backward, which can achieve a precise localization with one attempt. In this study, RACP could immediately decrease the blood pressure to target level, unlike SNP $(P=0.003)$. Furthermore, the aortic pressure could be rapidly restored to the physiological level before the pacing once RACP was stopped. Compared with the SNP group, the restoration time in the RACP group was significantly shorter $(P<0.001)$. In complicated cases requiring a second graft or postdilation, RACP can be applied again immediately. This enables the operator to solve the problem immediately rather than waiting for SNP to take effect, which is very important for preventing complications and shortening the duration of the total procedure. In the present study, the stent grafts were more precisely localized and deployed in the RACP group than in the SNP group $(P<0.001)$. It has been determined that whether the stent graft is deployed precisely correlates to the incidence of endoleak. ${ }^{4,8}$ Therefore, RACP might reduce the risk of endoleak by facilitating more precise stent graft deployment. However, we found no significant difference in the incidence of endoleak between the RACP and SNP groups, which might have resulted from the small sample size in our study. In the future, we plan to recruit more patients to see if there is any further difference between RACP and SNP groups.

Temporary percutaneous pacemaker implantation is a common therapy for bradycardia. When the heart beat amounts to 160 200 bpm, disharmonious systole begins to occur and left ventricle filling time is significantly reduced. 
These two factors remarkably reduce the cardiac output and also reduce the mean arterial pressure, to $30 \sim 60 \mathrm{mmHg} .{ }^{12}$ During TEVAR, the pacing frequency can be adjusted from 160 to $200 \mathrm{bpm}$ to reduce the aortic pressure to $30 \sim 60 \mathrm{mmHg}$ and completely eliminate pulse pressure, which keeps a relatively stable pressure in the aorta and thus facilitates the precise stent graft localization/deployment. Aortic pressure was significantly lower in the RACP group compared with the SNP group, but the risk of cognitive dysfunction, renal dysfunction, and paraplegia/hemiplegia in the RACP group was not higher than in the SNP group, probably due to the much shorter-duration hypotension and the faster restoration of blood pressure after RACP was stopped. Semmens et al demonstrated that long-lasting hypotension could result in low perfusion of the spinal cord and thus increase the risk of ischemic injury. In the present study, the duration of hypotension was significantly shorter in the RACP group when compared to the SNP group.

Common complications of RACP are mainly puncturesite-related (eg, puncture site hematoma and arteriovenous fistula $)^{13}$ and can be greatly reduced when RACP is performed by skilled doctors. Implanting the pacing lead under fluoroscopy and applying RACP for less than 10 seconds could reduce the incidence of complications to an acceptable level. In addition to puncture-related complications, RACP can cause tachycardia and bradycardia as well. In patients without established heart diseases or previous tachycardia, the likelihood of acquired tachycardia is less than $1 \%{ }^{14}$ In patients with left bundle branch block, occurrences of pacemaker dependency should be expected; a permanent pacemaker is needed in some cases. In the present study, there were three cases of left bundle branch block in the RACP group, yet we found no pacemaker dependency in these cases.

In an attempt to achieve precise deployment, some improvements in stent graft design have been made. The windsock effect was still detected in the present study, even with use of the new generation of stent graft. RACP can be considered an effective, stable, safe method with which to facilitate precise deployment of stent graft and should be routinely applied during TEVAR for Stanford B aortic dissection. In the future, we will perform long-term follow-up studies to evaluate any potential differences between RACP and SNP control of blood pressure during TEVAR.

\section{Conclusion}

During TEVAR for Stanford B aortic dissection, RACP was compared to medication-induced hypotension and was shown to be able to facilitate precise graft localization/deployment, without increasing the risk of complications.

\section{Author contributions}

JC, WH, SL: study conception and design, perform the study, data analysis and interpretation, drafting of the paper; DY, ZX, JL: study design, data analysis and interpretation, drafting of the paper.

\section{Disclosure}

The authors report no conflicts of interest in this work.

\section{References}

1. Xue L, Luo J, Mai J, Liu X. Change trend of clinical characteristics of aortic dissection over 10 years. Chin J Cardiol. 2007; 35(1):47-50. Chinese.

2. Farber MA, Criado FJ. Endovascular repair of nontraumatic ruptured thoracic aortic pathologies. Ann Vasc Surg. 2005;19:167-171.

3. Dorros G, Cohn JM. Adenosine-induced transient cardiac asystole enhances precise deployment of stent-grafts in the thoracic or abdominal aorta. J Endovasc Surg. 1996;3:270-272.

4. Bernard EO, Schmid ER, Lachat ML, Germann RC. Nitroglycerin to control blood pressure during endovascular stent-grafting of descending thoracic aortic aneurysms. J Vasc Surg. 2000;31:790-793.

5. Folstein MF, Folstein SE, McHugh PR. "Mini-mental state". A practical method for grading the cognitive state of patients for the clinician. J Psychiatr Res. 1975;12:189-198.

6. Grace J, Nadler JD, White DA, et al. Folstein vs modified Mini-Mental State Examination in geriatric stroke. Stability, validity, and screening utility. Arch Neurol. 1995;52:477-484.

7. Tombaugh TN, McIntyre NJ. The mini-mental state examination: a comprehensive review. J Am Geriatr Soc. 1992;40:922-935.

8. Nienaber CA, Kische S, Rehders TC, et al. Rapid pacing for better placing: comparison of techniques for precise deployment of endografts in the thoracic aorta. J Endovasc Ther. 2007;14:506-512.

9. Moon MC, Dowdall JF, Roselli EE. The use of right ventricular pacing to facilitate stent graft deployment in the distal aortic arch: a case report. J Vasc Surg. 2008;47:629-631.

10. Leurs LJ, Bell R, Degrieck Y, Thomas S, Hobo R, Lundbom J; EUROSTAR; UK Thoracic Endograft Registry collaborators. Endovascular treatment of thoracic aortic diseases: combined experience from the EUROSTAR and United Kingdom Thoracic Endograft registries. J Vasc Surg. 2004;40:670-679; discussion 679-680.

11. Kahn RA, Marin ML, Hollier L, Parsons R, Griepp R. Induction of ventricular fibrillation to facilitate endovascular stent graft repair of thoracic aortic aneurysms. Anesthesiology. 1998;88:534-536.

12. Pornratanarangsi S, Webster MW, Alison P, Nand P. Rapid ventricular pacing to lower blood pressure during endograft deployment in the thoracic aorta. Ann Thorac Surg. 2006;81:e21-e23.

13. Knight BP, Gersh BJ, Carlson MD, et al; American Heart Association Council on Clinical Cardiology (Subcommittee on Electrocardiography and Arrhythmias); Quality of Care and Outcomes Research Interdisciplinary Working Group; Heart Rhythm Society; AHA Writing Group. Role of permanent pacing to prevent atrial fibrillation: science advisory from the American Heart Association Council on Clinical Cardiology (Subcommittee on Electrocardiography and Arrhythmias) and the Quality of Care and Outcomes Research Interdisciplinary Working Group, in collaboration with the Heart Rhythm Society. Circulation. 2005;111:240-243.

14. Lee WA, Martin TD, Gravenstein N. Partial right atrial inflow occlusion for controlled systemic hypotension during thoracic endovascular aortic repair. J Vasc Surg. 2008;48:494-498. 
Clinical Interventions in Aging

Dovepress

\section{Publish your work in this journal}

Clinical Interventions in Aging is an international, peer-reviewed journal focusing on evidence-based reports on the value or lack thereof of treatments intended to prevent or delay the onset of maladaptive correlates of aging in human beings. This journal is indexed on PubMed Central, MedLine, the American Chemical Society's 'Chemical Abstracts

Service' (CAS), Scopus and the Elsevier Bibliographic databases. The manuscript management system is completely online and includes a very quick and fair peer-review system, which is all easy to use. Visit $\mathrm{http} / / / \mathrm{www}$.dovepress.com/testimonials.php to read real quotes from published authors.

Submit your manuscript here: http://www.dovepress.com/clinical-interventions-in-aging-journal 